\title{
Post hoc analysis of fecal samples from responders and non- responders to Lactobacillus reuteri DSM 17938 intervention
}

\author{
Henryk Szymański ${ }^{\square}$, Piotr Młynarz², Badr Qasem², Agnieszka Korzeniowska-Kowal², \\ Bogumiła Szponar³, Małgorzata Kałwak-Baran¹ and Hania Szajewska ${ }^{4}$
}

1Department of Paediatrics, St Hedwig of Silesia Hospital, Trzebnica, Poland; ${ }^{2}$ Department of Bioorganic Chemistry, Faculty of Chemistry, University of Science and Technology, Wrocław, Poland; ${ }^{3}$ Department of Immunology of Infectious Diseases, Hirszfeld Institute of Immunology and Experimental Therapy, Polish Academy of Sciences, Wroclaw, Poland; ${ }^{4 T h e}$ Medical University of Warsaw, Department of Paediatrics, Warsaw, Poland

We compared fecal samples from responders and nonresponders to administration of Lactobacillus reuteri DSM 17938. Data for this post hoc analysis were collected from an RCT assessing the efficacy of $L$. reuteri for the management of acute gastroenteritis. Responders were defined as subjects with diarrhea lasting no longer than 48 h. 44 children (17 responders and 27 non-responders) were analyzed. There were no differences in clinical characteristics and gut colonization between both groups. In the responder group, there were significantly lower levels of five metabolites before beginning of the intervention: lactate, choline, ethanol, creatine, and formate. The fecal calprotectin level did not differ between groups prior to the intervention, but its level was significantly lower after intervention in the responder group. Possibly, the responder group with a "metabolic niche", including lower level of metabolites, especially lactate, that are potential products of Lactobacillus genus, would determine the response to probiotic treatment. These findings need to be confirmed, but identification of some differences in the fecal metabolomics and the calprotectin level suggests that further studies are warranted.

Key words: probiotics, diarrhea, responders, metabolome, colonization

Received: 17 April, 2020; revised: 25 July, 2020; accepted: 27 July, 2020; available on-line: 18 September, 2020

$\square$ e-mail: henryk.t.szymanski@gmail.com

Funding. BioGaia provided the study products; the manufacturer had no role in the conception, design, or performance of this study, or in the analysis or interpretation of the data. There was no external funding. Funding of PM and BQ studies was supported by Politechnika Wrocławska (Grant No. Statutory Research 2019). Trial registration: ClinicalTrials.gov (NCT02989350).

Abbreviations: $\mathrm{CFU}$, colony forming unit: $\mathrm{Cl}$, confidence interval; ISAPP, International Scientific Association for Probiotics and Prebiotics; ITT, intention-to-treat analysis; MALDI-TOF MS, matrix-assisted laser desorption/ionization time-of-flight mass spectrometry $\mathrm{MD}$, mean difference; NMR, nuclear magnetic resonance; $\mathrm{RCT}$, randomized control trial

\section{INTRODUCTION}

There is interest in determining differences between responders and non-responders to probiotic interventions (Reid et al., 2010). Many factors may contribute to the effects of probiotics, including diet, baseline microbiota, dose, matrix, manufacturing conditions, use of medications (e.g., gut microbiota modifiers, such as antibiotics), and diarrhea etiology/vaccination status. Based on animal and human experiments, some recent studies suggest that probiotic administration (or at least combination of 11 strains used by the investigators) does not consistently change the gut microbiota composition and that individual responses to probiotic administration differ. It was also suggested that probiotic effects or lack of them may differ depending on the indigenous microbiota and gene-expression profiles (Suez et al., 2018; Zmora et al., 2018).

In line with many current guidelines, use of probiotics with documented efficacy may be considered in the management of acute gastroenteritis (Guarino et al., 2014, 2018). A 2016 meta-analysis of three randomized controlled trials $(n=256)$ found that when compared to a placebo or no treatment, the administration of Lactobacillus reuteri DSM 17938 (L. reuteri) has significantly reduced duration of diarrhea and increased the cure rate on day 1 and day 2 (Urbańska et al., 2016). However, Szymański and Szajewska found that $L$. reuteri did not reduce the duration of diarrhea when compared to a placebo as an adjunct to rehydration therapy, but it did reduce the duration of hospitalization. That study was a randomized, double-blind, placebo-controlled trial performed with children who had acute gastroenteritis (Szymański \& Szajewska, 2019). In this post hoc analysis, we compared the fecal properties of responders and non-responders to the administration of $L$. reuteri in participants of the previous trial.

\section{MATERIALS AND METHODS}

This post hoc analysis examined fecal samples from responders and non-responders to a probiotic intervention. Data were collected from a randomized, double-blind, placebo-controlled trial that evaluated the effectiveness of $L$. reuteri for the management of acute gastroenteritis in children. Written informed consent was obtained from parents or legal guardians before enrollment. All research was performed in accordance with relevant guidelines.

Full information about this study has been described in detail elsewhere (Szymański \& Szajewska, 2019). In brief, the trial included 100 children younger than 5 years of age with acute diarrhea lasting no more than 5 days. The children received $L$. reuteri at a dose of $2 \times 10^{8}$ colony-forming units (CFU)/day or a placebo for 5 consecutive days as a supplement to standard rehydration therapy. Of the 100 randomized children, 91 were included in the intention-to-treat analysis (ITT) (L. reuteri $n=44$, placebo $n=47)$. The duration of diarrhea after inclusion in the study was similar in both groups $(p=0.6)$. The groups were also similar with respect to all second- 
ary outcome measures with one exception. Compared with the placebo group, patients in the $L$. reuteri group had a shorter duration of hospitalization $(p=0.048)$. Adverse events were similar in both groups.

In this post hoc analysis, only responders and nonresponders in the $L$. reuteri group $(n=44)$ were compared. In accordance with recommendations regarding outcomes in the literature (Karas et al., 2015), responders were defined as the subjects with diarrhea lasting no longer than $48 \mathrm{~h}$. The subjects with diarrhea lasting more than $48 \mathrm{~h}$ were defined as non-responders. The following fecal analyses were performed: assessment of the colonization of the gastrointestinal tract by L. reuteri, metabolomic analysis, and assessment of the calprotectin level. Calprotectin is produced by white blood cells and is used as an indicator of the severity of inflammation of the intestine (Burri \& Beglinger, 2014).

Sample collection. Fecal samples (at least $1 \mathrm{~g}$ ) were obtained on day 1 (prior to the intervention) and on day 8 (after the intervention). Stool was collected from all children at the start of the study because it was unpredictable as to who would or would not respond to the intervention. However, only data from responders and non-responders in the group treated with $L$. reuteri are presented here. Two samples were obtained from the majority of participants in the study. However, some samples were unsuitable for evaluation because the amount of material collected was too small, and therefore in the end only 19 samples were taken for metabolomic analysis and 21 for calprotectin analysis.

Samples for the assessment of the gastrointestinal tract colonization by $L$. reuteri were collected immediately after stool output by the child. The samples were obtained using a transport tube with Amies transport medi$\mathrm{um}$ and then stored at +2 to $+8^{\circ} \mathrm{C}$. After transfer to the laboratory, samples were plated on the MRS Broth PS60, which is a selective medium for isolation of lactic acid bacteria. The growing strains were then identified using matrix-assisted laser desorption/ionization time-of-flight mass spectrometry (MALDI-TOF MS). The samples used for metabolomic analysis and the determination of fecal calprotectin levels were stored at $-20^{\circ} \mathrm{C}$ until the tests were performed.

Microorganism identification by the MALDITOF MS Biotyper method. For the MALDI-TOF MS analysis, two to five colonies of actively growing cultures were incubated for $48 \mathrm{~h}$ on the MRS agar at $37^{\circ} \mathrm{C}$ under both, the aerobic and anaerobic conditions, and then suspended in $300 \mu \mathrm{l}$ of double-distilled water. Next, $900 \mu \mathrm{l}$ of absolute ethanol were added. The sample was centrifuged two times $(13000 \times \mathrm{g}, 3 \mathrm{~min})$, and the sediment was dried at room temperature. Lysates were prepared by adding $50 \mu \mathrm{l}$ of $70 \%$ formic acid to the bacterial pellet, mixing thoroughly, adding $50 \mu \mathrm{l}$ of acetonitrile, and mixing the sample again.

After centrifugation $(13000 \times g, 2 \mathrm{~min})$, the supernatant was transferred to a fresh tube, and $1 \mu \mathrm{l}$ of the bacterial protein lysate was applied to a 384 ground steel MALDI target plate (Bruker Daltonics, Bremen, Germany) and air-dried at room temperature. The sample was then overlaid with $1 \mu$ of $\alpha$-cyano-4-hydroxycinnamic acid matrix solution (HCCA; Bruker Daltonics) and air-dried again. The measurements were performed using a Bruker Daltonics UltrafleXtreme spectrometer. Spectra were recorded in a positive linear mode for a mass range of 2000 to $20000 \mathrm{Da}$ (laser frequency $200 \mathrm{~Hz}$; ion source voltage one, $25 \mathrm{kV}$; ion source voltage two, $23.5 \mathrm{kV}$; lens voltage, $6.0 \mathrm{kV}$ ).
Each spectrum was obtained by averaging 1500 laser shots that were acquired from three spot positions under the control of flexControl software 3.1 (Bruker Daltonics). The spectra were externally calibrated using an E. coli DH5-alpha standard (Bruker Daltonics). The calibrant consisted of six ribosomal proteins from E. coli with added RNAse $A$ and myoglobin to cover a range of 3637.8 to 16952.3 Da. Biotyper 3.1 software (Bruker Daltonics) and a database containing 4613 entries were used for identification. According to the manufacturer, the following score values were used: less than 1.7, identification not reliable; $1.7-2.0$, probable genus identification; 2.0-2.3, secure genus identification and probable species identification; and more than 2.3 , highly probable species identification (van Belkum et al., 2015).

Fecal metabolomic analysis. Before performing the analysis, the samples were thawed at room temperature and then transferred to plastic tubes, weighed, and lyophilized. After this process, dry feces were weighed again, and methanol extracts of metabolites were obtained. Next, $59 \pm 22 \mathrm{mg}$ of feces were placed in plastic Eppendorf tubes. After adding $1 \mathrm{ml}$ of methanol and two steel balls to crush and homogenize the feces, the samples were shaken in a Thermo Mixer at room temperature for $10 \mathrm{~min}$. Next, the specimens were centrifuged at $12500 \mathrm{rpm}$ at room temperature for $10 \mathrm{~min}$, and then the supernatant was collected and evaporated using a Speedvac instrument.

The dry mass was kept at $-80^{\circ} \mathrm{C}$ until the analysis was carried out. Nuclear magnetic resonance (NMR) spectroscopy was used to analyze the metabolites in the fecal samples. Prior to the NMR analysis, the samples were dissolved in $600 \mu \mathrm{L}$ of PBS $\left(0.5 \mathrm{M}, \mathrm{pH}=7.00,50 \% \mathrm{D}_{2} \mathrm{O}\right.$, containing $\mathrm{NaN}_{3}$ and $3 \mathrm{mM}$ TSP), vortexed, and centrifuged $\left(12000 \times \mathrm{rpm}, 10 \mathrm{~min}, 4^{\circ} \mathrm{C}\right)$. At the end, $550 \mu \mathrm{L}$ of supernatant were collected into a $5-\mathrm{mm}$ NMR tube (SP, $5 \mathrm{~mm}$ ARMAR Chemicals) for measurement.

All NMR spectra were recorded using a Bruker 600 $\mathrm{MHz}$ AVANCE II spectrometer with a zgesgp pulse sequence, a relaxation delay of $3.5 \mathrm{~s}$, a time domain of $65 \mathrm{k}$, and a spectral width of $15 \mathrm{ppm}$. Manual phase correction was performed using Topspin 3.2 software (Bruker, GmBH, Germany), and baseline correction was performed using MestReNova 12.0.4 software (Mestrelab Research, S.L., Spain). All NMR spectra were referenced to the TSP resonance $(\delta=0.000 \mathrm{ppm})$.

The resonance signals were aligned using the correlation optimized warping algorithm (COW) (Tomasi et al., 2004), and icoshift was performed in Matlab (v 8.3, Mathworks Inc.) (Savorani et al., 2010). Regions of the spectrum containing residual water and TSP were excluded from calculations. Normalization was performed for all of the spectra using the Probabilistic Quotient Normalization (PQN) method (Dieterle et al., 2006). Metabolite resonances were identified using online databases (Biological Magnetic Resonance Data Bank, Human Metabolome Data Base) and assignments published in the literature.

Fecal calprotectin. The calprotectin ELISA test (EUROIMMUN) was used to assess fecal calprotectin levels. Measurements were performed according to manufacturer's instructions.

Statistical analysis. Nominal variables are presented as $n(\%)$, while continuous variables are expressed as the mean or median with the $95 \%$ confidence interval (CI) depending on the distribution of the data. Data were tested for normality using the Shapiro-Wilk test. For nominal data, significant differences between groups were detected by conducting a group comparison us- 
ing the chi-squared test or Fisher exact test. An independent-samples $t$-test (student's $t$-test) or nonparametric Mann-Whitney $U$ test were used as appropriate for continuous data. Additionally, differences in means or medians, depending on the data distribution, were calculated for continuous variables with 95\% CIs. All tests were two-tailed, and differences were considered significant at the level of $p<0.05$. All of the analyses were carried out using the statistical software $\mathrm{R}$ version 3.4 .4 (http:// cran.r-project.org).

Ethics. The Ethics Committee of the Lower Silesia Medical Chamber approved this study (1\&2/ $\mathrm{PB} / 2016)$. The trial was registered at ClinicalTrials.gov (NCT02989350).

\section{RESULTS}

A total of 44 children (median age: 20 months, 95\% CI: $12.0 ; 34.2 ; 24$ boys/20 girls) treated with $L$. renteri were included in the analysis. Among them, there were 17 responders and 27 non-responders.

\section{Clinical characteristics}

There were no significant differences in any of the clinical characteristics between groups except that there was a higher percentage of females in the responder group than the non-responder group $(64.7 \%$ vs. $33.3 \%$, $p=0.04$ ) (Table 1). When compared with the non-responder group, there were no significant differences in the responder group in either the duration of diarrhea before enrollment $(p=0.57)$ or the severity of diarrhea assessed using the modified Vesikari scale $(p=0.73)$.

\section{Colonization}

Colonization of the gastrointestinal tract with $L$. reuteri was confirmed in $14(31.8 \%)$ of the 44 subjects treated with $L$. reuteri. However, there was no significant difference between the responders and non-responders in the percentage of subjects with colonization (8/17 [47\%] versus $6 / 27$ [22.2\%], respectively, $p=0.7)$.

\section{Fecal metabolomic analysis}

For the metabolomic analyses, stool samples from only 19 participants were available. Some samples were not provided by caregivers and some of them were not suitable for analysis.
There were no significant differences between the responders $(n=8)$ and non-responders $(n=11)$ with a few exceptions. Levels of five metabolites were significantly lower in the responder group before onset of the intervention: lactate, choline, ethanol, creatine, and formate (Table 2). However, there were no significant differences between the responder and non-responder groups in the levels of any of the metabolites after intervention ( $\mathrm{Ta}$ ble 3).

\section{Fecal calprotectin}

The fecal calprotectin level did not differ between groups prior to the intervention $(p=0.46)$. However, after the intervention, there was a significantly lower level of fecal calprotectin in the responder group (MD $213.70 \mu \mathrm{g} / \mathrm{g}$ feces, 95\% CI 25.90 to $443.62 ; p=0.03$ ) (Table 4). In the responder group, there was a significant decrease in the calprotectin level after the $L$. reuteri intervention when compared to the pre-intervention level (MD - 708.9 $\mu \mathrm{g} / \mathrm{g}$ feces, 95\% CI - 1767; - 71.3; $p=0.016)$.

\section{DISCUSSION}

\section{Principal findings}

The aim of this study was to compare properties of fecal samples from responders and non-responders to administration of $L$. reuteri. Data for this post hoc analysis were collected from a randomized, double-blind, placebo-controlled trial that evaluated the effectiveness of $L$. reuteri for the management of acute gastroenteritis in children. There were no significant differences in clinical characteristics between responders and non-responders except that the response was more likely to occur in females. Colonization of the gastrointestinal tract with L. reuteri was similar in responders and non-responders. However, colonization was confirmed in less than onethird of subjects treated with $L$. reuteri.

Notably, colonization is not needed for probiotics to exert their beneficial effects, and other mechanisms, such as production of active compounds, may play an important role here (Talarico et al., 1988). There were no significant differences in the fecal metabolite levels between groups with a few exceptions. In the responder group, there were significantly lower levels of five metabolites

Table 1. Clinical characteristics of non-responders and responders in subjects treated with L. reuteri before onset of the intervention.

\begin{tabular}{lllc}
\hline Characteristics & Non-responders & Responders & $p^{1}$ \\
\hline$n$ & 27 & 17 & $6 / 11$ \\
\hline Gender (boys/girls) & $18 / 9$ & $23.7(16.3 ; 36.3)$ & 0.37 \\
\hline Age, mo, median $(95 \% \mathrm{Cl})$ & $19.4(15.5 ; 26.0)$ & $10.1(9.0 ; 11.2)$ & 0.73 \\
\hline Vesikari scale, score, mean $(95 \% \mathrm{Cl})$ & $10.3(9.4 ; 11.2)$ & $43.9(32.4 ; 55.4)$ & 0.57 \\
\hline Duration of diarrhea before enrollment, $\mathrm{h}$, mean $(95 \% \mathrm{Cl})$ & $48.2(37.1 ; 59.4)$ & $16(94.1)$ & $>0.99$ \\
\hline Vomiting, $\mathrm{n}(\%)$ & $24(88.9)$ & $8(47.1)$ & 0.22 \\
\hline Fever $>38^{\circ} \mathrm{C}, \mathrm{n}(\%)$ & $18(66.7)$ & $0(0)$ & 0.15 \\
\hline Bloody stools, $\mathrm{n}(\%)$ & $4(14.8)$ & $7(41.2)$ & 0.98 \\
\hline Rotavirus, $\mathrm{n}(\%)$ & $11(40.7)$ & $0(0)$ & $>0.99$ \\
\hline Adenovirus, $\mathrm{n}(\%)$ & $0(0)$ & \\
\hline
\end{tabular}

'Student's $t$-test, Mann-Whitney $U$ test, Chi-squared test, or Fisher exact test as appropriate 
Table 2. Metabolite levels before onset of the intervention in non-responders and responders in subjects treated with $L$. reuteri (data available for 19 out of 44 subjects).

\begin{tabular}{|c|c|c|c|}
\hline Metabolites & Non-responders ${ }^{1}$ & Responders $^{1}$ & $p^{2}$ \\
\hline$n$ & 11 & 8 & \\
\hline Formate & $0.06(0.04 ; 0.18)$ & $0.03(0.02 ; 0.13)$ & 0.028 \\
\hline Unknown & $0.06(0.03 ; 0.09)$ & $0.06(0.01 ; 0.12)$ & 0.92 \\
\hline Xanthine & $0.07(0.04 ; 0.09)$ & $0.09(0.05 ; 0.13)$ & 0.23 \\
\hline Uracil & $0.21(0.09 ; 0.34)$ & $0.29(0.18 ; 0.40)$ & 0.33 \\
\hline Phenyloalanine & $0.56(0.31 ; 0.80)$ & $0.44(0.24 ; 0.63)$ & 0.43 \\
\hline Tyrosine & $0.25(0.17 ; 0.33)$ & $0.24(0.14 ; 0.34)$ & 0.83 \\
\hline Lactate & $3.32(1.99 ; 6.95)$ & $0.61(0.39 ; 4.08)$ & 0.016 \\
\hline Creatine & $0.08(0.02 ; 0.14)$ & $0.01(0.00 ; 0.02)$ & 0.022 \\
\hline Methanol & $1.20(0.61 ; 43.96)$ & $0.58(0.33 ; 11.8)$ & 0.4 \\
\hline Choline & $1.34(0.79 ; 1.90)$ & $0.28(0.09 ; 0.47)$ & 0.002 \\
\hline Trimethyloamine & $0.03(0.02 ; 0.49)$ & $0.08(0.02 ; 0.23)$ & 0.97 \\
\hline Succinate & $0.67(0.42 ; 3.78)$ & $1.31(0.59 ; 6.56)$ & 0.44 \\
\hline 4-aminobutyrate & $0.33(0.19 ; 0.69)$ & $0.42(0.22 ; 0.75)$ & 0.49 \\
\hline Propionate & $0.57(0.33 ; 0.80)$ & $0.89(0.42 ; 1.36)$ & 0.14 \\
\hline Methionine & $0.28(0.11 ; 0.45)$ & $0.31(0.14 ; 0.48)$ & 0.78 \\
\hline Unknown & $0.24(0.21 ; 1.77)$ & $0.22(0.16 ; 0.55)$ & 0.18 \\
\hline Acetate & $5.99(3.51 ; 10.64)$ & $6.88(3.60 ; 13.67)$ & 0.84 \\
\hline Alanine & $2.50(1.93 ; 5.10)$ & $5.6(2.86 ; 9.00)$ & 0.15 \\
\hline Metylomalonate & $0.18(0.13 ; 0.60)$ & $0.11(0.08 ; 0.19)$ & 0.13 \\
\hline Ethanol & $0.21(0.11 ; 0.31)$ & $0.06(0.03 ; 0.09)$ & 0.007 \\
\hline Isobutyrate & $0.88(0.41 ; 0.35)$ & $0.79(0.48 ; 1.10)$ & 0.75 \\
\hline Valine & $1.07(0.51 ; 0.64)$ & $1.02(0.51 ; 1.53)$ & 0.88 \\
\hline Isoleucine & $1.80(0.85 ; 2.76)$ & $1.58(0.89 ; 2.27)$ & 0.72 \\
\hline Leucine & $0.07(0.24 ; 0.90)$ & $0.12(0.08 ; 0.24)$ & 0.18 \\
\hline Butyrate & $0.55(0.24 ; 0.87)$ & $0.84(0.38 ; 1.30)$ & 0.24 \\
\hline
\end{tabular}

1Values are means $(95 \% \mathrm{Cl})$ or medians $(95 \% \mathrm{Cl})$. ${ }^{2}$ Groups were compared with the use of the Mann-Whitney $\mathrm{U}$ test or Student's $t$-test for continuous variables

(lactate, choline, ethanol, creatine, and formate) before beginning of the intervention. The fecal calprotectin level did not differ between groups prior to the intervention. However, there was a significantly lower level of fecal calprotectin after the intervention in the responder group. Additionally, responders showed a significant reduction in the fecal calprotectin level after the intervention when compared to the pre-intervention level.

\section{Strengths and limitations}

The strengths and limitations of the original trial were discussed in our earlier publication (Szymański \& Szajewska, 2019). In brief, the strengths included the methodology: the study protocol was published prior to the enrollment of the first subject, there was adequate generation of the allocation sequence and allocation concealment, there was adequate blinding, and an intention-totreat analysis was performed. One of the limitations is that there was no formal confirmation of the identity of the organism or the number of CFUs in the study product. However, in a parallel study (Kołodziej \& Szajew- ska, 2019), both study products were blindly tested using genetic methods, and the active product was confirmed to contain $L$. reuteri DSM 17938.

One limitation of the current responder/non-responder analysis is the post hoc design, which precludes robust conclusions. However, it does allow generation of hypotheses for future studies (Curran-Everett \& Milgrom, 2013). Additionally, responders and non-responders were not predefined. It may be argued that defining responders as those with diarrhea lasting no longer than $48 \mathrm{~h}$ is not optimal. However, according to the literature the proportion of patients who recover by $48 \mathrm{~h}$ is one of five outcomes for therapeutic studies (Karas et al., 2015). Another limitation is the small number of subjects available for analysis. However, the sample size was calculated for the primary outcome of the original trial. Thus, caution is needed when interpreting findings of this post hoc analysis.

Despite limitations of the post hoc analysis, we decided to perform it since there is interest in the question of why some subjects respond to probiotic interventions 
Table 3. Metabolite levels after the intervention in non-responders and responders in subjects treated with $L$. reuteri (data available for 19 out of 44 subjects).

\begin{tabular}{|c|c|c|c|}
\hline Metabolites & Non-responders ${ }^{1}$ & Responders $^{1}$ & $p^{2}$ \\
\hline$n$ & 11 & 8 & \\
\hline Formate & $0.04(0.03 ; 0.23)$ & $0.02(0.02 ; 0.16)$ & 0.35 \\
\hline Unknown & $0.06(0.03 ; 0.10)$ & $0.05(0.03 ; 0.07)$ & 0.73 \\
\hline Xanthine & $0.09(0.06 ; 0.11)$ & $0.10(0.07 ; 0.13)$ & 0.44 \\
\hline Uracil & $0.22(0.16 ; 0.28)$ & $0.26(0.18 ; 0.34)$ & 0.35 \\
\hline Phenyloalanine & $0.33(0.24 ; 0.42)$ & $0.34(0.21 ; 0.46)$ & 0.88 \\
\hline Tyrosine & $0.22(0.15 ; 0.28)$ & $0.25(0.15 ; 0.36)$ & 0.51 \\
\hline Lactate & $0.70(0.52 ; 2.28)$ & $0.43(0.28 ; 2.18)$ & 0.21 \\
\hline Creatine & $0.02(0.01 ; 0.04)$ & $0.03(-0.00009 ; 0.06)$ & 0.63 \\
\hline Methanol & $5.69(1.29 ; 39.06)$ & $17.79(4.59 ; 44.29)$ & 0.24 \\
\hline Choline & $0.18(0.12 ; 0.27)$ & $0.17(0.07 ; 0.46)$ & 0.84 \\
\hline Trimethyloamine & $0.10(0.04 ; 0.15)$ & $0.05(0.01 ; 0.08)$ & 0.15 \\
\hline Succinate & $0.66(0.33 ; 2.42)$ & $0.54(0.24 ; 4.86)$ & 0.97 \\
\hline 4-aminobutyrate & $0.21(0.09 ; 0.47)$ & $0.05(0.03 ; 0.22)$ & 0.11 \\
\hline Propionate & $1.08(0.84 ; 1.99)$ & $1.27(0.66 ; 2.44)$ & 0.66 \\
\hline Methionine & $0.22(0.17 ; 0.27)$ & $0.23(0.11 ; 0.36)$ & 0.86 \\
\hline Unknown & $0.41(0.31 ; 1.22)$ & $0.45(0.26 ; 1.11)$ & 0.92 \\
\hline Acetate & $10.71(7.12 ; 14.29)$ & $11.81(7.66 ; 15.97)$ & 0.65 \\
\hline Alanine & $3.44(2.29 ; 4.59)$ & $3.34(2.02 ; 4.65)$ & 0.89 \\
\hline Metylomalonate & $0.14(0.11 ; 0.32)$ & $0.14(0.07 ; 0.40)$ & 0.49 \\
\hline Ethanol & $0.05(0.04 ; 0.07)$ & $0.04(0.03 ; 0,07)$ & 0.23 \\
\hline Isobutyrate & $0.60(0.41 ; 0.78)$ & $0.56(0.29 ; 0.84)$ & 0.81 \\
\hline Valine & $0.83(0.57 ; 1.10)$ & $0.80(0.41 ; 1.19)$ & 0.87 \\
\hline Isoleucine & $1.16(0.81 ; 1.52)$ & $1.11(0.64 ; 1.57)$ & 0.82 \\
\hline Leucine & $0.10(0.08 ; 0.12)$ & $0.10(0.05 ; 0.16)$ & 0.86 \\
\hline Butyrate & $1.14(0.68 ; 2.08)$ & $1.13(0.44 ; 2.41)$ & 0.97 \\
\hline
\end{tabular}

VValues are means $(95 \% \mathrm{Cl})$ or medians $(95 \% \mathrm{Cl}) .{ }^{2}$ Groups were compared with the use of the Mann-Whitney U test or Student's $t$-test for continuous variables

Table 4. Comparison of the calprotectin level ( $\mu \mathrm{g} / \mathrm{g}$ feces) between groups prior to the intervention and after the intervention.

\begin{tabular}{llll}
\hline & Non-responders & Responders & $p$ \\
\hline$n$ & 13 & 8 & $762.1(106.3 ; 1890.2)$ \\
\hline Calprotectin before the intervention & $206.2(114.3 ; 1397.1)$ & $53.3(10.8 ; 321.0)$ & 0.46 \\
\hline Calprotectin after the intervention & $267.0(166.2 ; 1026.4)$ & 0.03 & \\
\hline
\end{tabular}

Values are medians $(95 \% \mathrm{Cl})$

while others do not. In 2010, the International Scientific Association for Probiotics and Prebiotics (ISAPP) addressed this issue (Reid et al., 2010). The ISAPP experts formulated four recommendations. First, the end goal of the study needs to be clearly defined. Second, the study design should "maximize the chance of a positive response by identifying precise parameters and defining the level of response that will be tested." Third, the selection of probiotic strains should be based on solid scientific criteria. Fourth, the study cohort should be selected carefully.
With respect to the fourth recommendation, the experts suggested that studies "use biological or genetic markers when available to stratify the patient population before enrollment and decide at what point the intervention will provide the best outcome (for example, in the acute phase of the disease, during remission, and with or without use of pharmaceutical agents)." Thus, the ISAPP experts indicated the importance of using metabolomics in clinical trials assessing probability of a positive response to treatment with a given probiotic.

In that sense, our study meets the ISAPP recommendations. However, as stated earlier, this post hoc analy- 
sis of responders and non-responders is underpowered for final conclusions. Nevertheless, further evaluation is warranted for metabolomics, which is defined as the "systematic identification and quantification of the small molecule metabolic products (the metabolome) of a biological system (cell, tissue, organ, biological fluid, or organism) at a specific point of time (Metabolomics - Latest Research and News | Nature, n.d.)."

In the context of previous studies, it allows to think that there is a "metabolic niche" and that lower level of metabolites, especially lactate, that are potential products of the Lactobacillus genus would determine the response to probiotic treatment (Shepherd et al., 2018). The metabolomics approach could be used in diagnosis and management of diseases involving the gastrointestinal tract (Dawiskiba et al., 2014; Lin et al., 2016; Zeber-Lubecka et al., 2016), cardiovascular system (Zordoky et al., 2015), and obstetrics (Miranda et al., 2018) (e.g., eclampsia (Kelly et al., 2017), as well as the study of cancer (Armitage \& Ciborowski, 2017; Kaushik \& DeBerardinis, 2018), neurodegenerative diseases (Botas et al., 2015), diabetes (Merino et al., 2018), and many others. Thus, the application of metabolomics instrumentation is one of the components of the development of personalized medicine (Jacob et al., 2019). Furthermore, metabolomics and genomics are the methods of choice for studying the microbiome interaction and its influence on the human organism (Holmes et al., 2012; Wang et al., 2019). As an example of the great potential of the metabolomics method, the American Heart Association has recently published a position paper describing the wide range of applications of metabolomics in cardiovascular health and disease, and determining directions for further research (Cheng et al., 2017). It is likely that other scientific societies will also address this issue.

\section{CONCLUSIONS}

This is the first study to attempt to characterize responders and non-responders to an $L$. reuteri intervention. While the findings of this post hoc analysis need to be confirmed, identification of some differences in the fecal metabolomics and calprotectin levels suggests that further studies are warranted. Identification of likely responders is needed to help guide the selection of subjects for successful therapies involving $L$. reuteri or other probiotics.

\section{Authors' contributions}

H. Szymański initially conceptualized this study. P. Młynarz, B. Qasem, A. Korzeniowska-Kowal, B. Szponar, and M. Kałwak-Baran were responsible for the analyses. H. Szymański was responsible for data collection. All authors were responsible for data analysis and interpretation. H. Szymański and H. Szajewska assumed the main responsibility for writing of the first draft of this manuscript. All authors contributed to and agreed upon the final version.

\section{Disclosure statement}

H. Szymański, P. Młynarz, B. Qasem, A. Korzeniowska-Kowal, B. Szponar, and M. Kałwak-Baran declare no conflicts of interest.

H. Szajewska has served as a speaker for BioGaia, the manufacturer of $L$. reuteri DSM 17938.

\section{REFERENCES}

Armitage EG, Ciborowski M (2017) Applications of metabolomics in cancer studies. In Advances in Experimental Medicine and Biology. York LLC eds, pp 209-234. Springer New. https://doi.org/10.1007/9783-319-47656-8 9

Botas A, Campbell HM, Han X, Maletic-Savatic M (2015) Metabolomics of Neurodegenerative Diseases. In International Review of Neurobiology. Michael J. Hurley ed, Vol 122, pp 53-80. Academic Press Inc. https://doi.org/10.1016/bs.irn.2015.05.006

Burri E, Beglinger C (2014) The use of fecal calprotectin as a biomarker in gastrointestinal disease. Expert Rev Gastroenterol Hepatol 8: 197-210. https://doi.org/10.1586/17474124.2014.869476

Cheng S, Shah SH, Corwin EJ, Fiehn O, Fitzgerald RL, Gerszten RE, Illig T, Rhee EP, Srinivas PR, Wang TJ, Jain M (2017) Potential impact and study considerations of metabolomics in cardiovascular health and disease: a scientific statement from the American Heart Association. Circulation: Cardiovascular Genetics 10: e000032. https:// doi.org/10.1161/HCG.0000000000000032

Curran-Everett D, Milgrom H (2013) Post-hoc data analysis: benefits and limitations. Curr Opin Allergy Clin Immunol 13: 223-224. https:// doi.org/10.1097/ACI.0b013e3283609831

Dawiskiba T, Deja S, Mulak A, Ząbek A, Jawień E, Pawełka D, Młynarz P (2014) Serum and urine metabolomic fingerprinting in diagnostics of inflammatory bowel diseases. World J Gastroenterol 20: 163-74. https://doi.org/10.3748/wig.v20.i1.163

Dieterle F, Ross A, Schlotterbeck G, Senn H (2006) Probabilistic quotient normalization as robust method to account for dilution of complex biological mixtures. Application in1H NMR metabonomics. Anal Chem 78: 4281-4290. https://doi.org/10.1021/ac051632c

Guarino A, Ashkenazi S, Gendrel D, Lo Vecchio A, Shamir R, Szajewska H (2014). European Society for Pediatric Gastroenterology, Hepatology, and Nutrition/European Society for Pediatric Infectious Diseases Evidence-Based Guidelines for the Management of Acute Gastroenteritis in Children in Europe. J Pediatric Gastroenterol Nutrit 59: 132-152. https://doi.org/10.1097/MPG.0000000000000375

Guarino A, Lo Vecchio A, Dias JA, Berkley JA, Boey C, Bruzzese D, Cohen MB, Cruchet S, Liguoro I, Salazar-Lindo E, Sandhu B, Sherman PM, Shimizu T (2018) Universal recommendations for the management of acute diarrhea in nonmalnourished children. $J$ Pediatric Gastroenterol Nutrit 67: 586-593. https://doi.org/10.1097/ MPG.0000000000002053

Holmes E, Li JV, Marchesi JR, Nicholson JK (2012) Gut microbiota composition and activity in relation to host metabolic phenotype and disease risk. Cell Metabolism 16: 559-564. https://doi. org/10.1016/j.cmet.2012.10.007

Jacob M, Lopata AL, Dasouki M, Abdel Rahman AM (2019) Metabolomics toward personalized medicine. Mass Spectrometry Rev 38: 221238. https://doi.org/10.1002/mas.21548

Karas J, Ashkenazi S, Guarino A, Vecchio ALo, Shamir R, Vandenplas Y, Szajewska H (2015) A core outcome set for clinical trials in acute diarrhoea. Arch Dis Childhood 100: 359-363. https://doi. org/10.1136/archdischild-2014-307403

Kaushik AK, DeBerardinis RJ (2018) Applications of metabolomics to study cancer metabolism. Biochim Biophys Acta - Rev Cancer 1870: 2-14. https://doi.org/10.1016/j.bbcan.2018.04.009

Kelly RS, Giorgio RT, Chawes BL, Palacios NI, Gray KJ, Mirzakhani H, Wu A, Blighe K, Weiss ST, Lasky-Su J (2017) Applications of metabolomics in the study and management of preeclampsia: a review of the literature. Metabolomics 13: 86. https://doi.org/10.1007/ s11306-017-1225-8

Kołodziej M, Szajewska H (2019) Lactobacillus reuteri DSM 17938 in the prevention of antibiotic-associated diarrhoea in children: a randomized clinical trial. Clin Microbiol Infect 25: 699-704. https://doi. org/10.1016/j.cmi.2018.08.017

Lin Y, Ma C, Liu C, Wang Z, Yang J, Liu X, Shen Z, Wu R (2016) NMR-based fecal metabolomics fingerprinting as predictors of earlier diagnosis in patients with colorectal cancer. Oncotarget 7: 29454 29464. https://doi.org/10.18632/oncotarget.8762

Merino J, Leong A, Liu CT, Porneala B, Walford GA, von Grotthuss M, Wang TJ, Flannick J, Dupuis J, Levy D, Gerszten RE, Florez JC, Meigs JB (2018) Metabolomics insights into early type 2 diabetes pathogenesis and detection in individuals with normal fasting glucose. Diabetologia 61: 1315-1324. https://doi.org/10.1007/ s00125-018-4599-x

Metabolomics - Latest research and news | Nature. (n.d.). Retrieved July 20, 2019, from https://www.nature.com/subjects/metabolomics

Miranda J, Simões RV, Paules C, Cañueto D, Pardo-Cea MA, GarcíaMartín ML, Crovetto F, Fuertes-Martin R, Domenech M, Gómez-Roig MD, Eixarch E, Estruch R, Hansson SR, Amigó N, Cañellas N, Crispi F, Gratacós E (2018) Metabolic profiling and targeted lipidomics reveals a disturbed lipid profile in mothers and fetuses with intrauterine growth restriction. Sci Rep 8: 1-14. https:// doi.org/10.1038/s41598-018-31832-5

Reid G, Gaudier E, Guarner F, Huffnagle GB, Macklaim JM, Munoz AM, Martini M, Ringel-Kulka T, Sartor BR, Unal RR, Verbeke K, 
Walter J (2010) Responders and non-responders to probiotic interventions: How can we improve the odds? Gut Microbes 1: 1-5. https://doi.org/10.4161/gmic.1.3.12013

Savorani F, Tomasi G, Engelsen SB (2010) icoshift: A versatile tool for the rapid alignment of $1 \mathrm{D}$ NMR spectra. I Magnetic Resonance 202. 190-202. https://doi.org/10.1016/j.jmr.2009.11.012

Shepherd ES, Deloache WC, Pruss KM, Whitaker WR, Sonnenburg JL (2018) An exclusive metabolic niche enables strain engraftment in the gut microbiota. Nature 557: 434-438. https://doi.org/10.1038/ s41586-018-0092-4

Suez J, Zmora N, Zilberman-Schapira G, Mor U, Dori-Bachash M, Bashiardes S, Zur M, Regev-Lehavi D, Ben-Zeev Brik R, Federici S, Horn M, Cohen Y, Moor AE, Zeevi D, Korem T, Kotler E, Harmelin A, Itzkovitz S, Maharshak N, Elinav E (2018) Post-antibiotic gut mucosal microbiome reconstitution is impaired by probiotics and improved by autologous FMT. Cell 174: 1406-1423. https:// doi.org/10.1016/j.cell.2018.08.047

Szymański H, Szajewska H (2019) Lack of efficacy of Lactobacillus reuteri DSM 17938 for the treatment of acute gastroenteritis. The Pediatric Infect Dis J 38: e237-e242. https://doi.org/10.1097/ INF.0000000000002355

Talarico TL, Casas IA, Chung TC, Dobrogosz WJ (1988) Production and isolation of reuterin, a growth inhibitor produced by Lactobacillus reuteri. Antimicrob Agents Chemother 32: 1854-1858. https://doi. org/10.1128/AAC.32.12.1854

Tomasi G, Van Den Berg F, Andersson C (2004) Correlation optimized warping and dynamic time warping as preprocessing methods for chromatographic data. J Chemometrics 18: 231-241. https://doi. org/10.1002/cem.859
Urbańska M, Gieruszczak-Białek D, Szajewska H (2016) Systematic review with meta-analysis: Lactobacillus renteri DSM 17938 for diarrhoeal diseases in children. Alimen Pharmacol Ther 43: 1025-1034. https://doi.org/10.1111/apt.13590

Wang Q, Wang K, Wu W, Giannoulatou E, Ho JWK, Li L (2019) Host and microbiome multi-omics integration: applications and methodologies. Biophys Rev 11: 55-65. https://doi.org/10.1007/ s12551-018-0491-7

Zeber-Lubecka N, Kulecka M, Ambrozkiewicz F, Paziewska A, Goryca K, Karczmarski J, Rubel T, Wojtowicz W, Mlynarz P, Marczak L, Tomecki R, Mikula M, Ostrowski J (2016) Limited prolonged effects of rifaximin treatment on irritable bowel syndrome-related differences in the fecal microbiome and metabolome. Gut Microbes 7: 397-413. https://doi.org/10.1080/19490976.2016.1215805

Zmora N, Zilberman-Schapira G, Suez J, Mor U, Dori-Bachash M, Bashiardes S, Kotler E, Zur M, Regev-Lehavi D, Brik RBZ, Federici S, Cohen Y, Linevsky R, Rothschild D, Moor AE, Ben-Moshe S, Harmelin A, Itzkovitz S, Maharshak N, Elinav E (2018) Personalized gut mucosal colonization resistance to empiric probiotics is associated with unique host and microbiome features. Cell 174: 1388-1405. e21. https://doi.org/10.1016/j.cell.2018.08.041

Zordoky BN, Sung MM, Ezekowitz J, Mandal R, Han B, Bjorndahl TC, Bouatra S, Anderson, T, Oudit GY, Wishart DS, Dyck JRB (2015) Metabolomic fingerprint of heart failure with preserved ejection fraction. PLOS ONE 10: e0124844. https://doi.org/10.1371/ journal.pone. 0124844 